\title{
Biopolítica y fuerza de trabajo. Virno lee a Foucault entre Aristóteles y Marx
}

\author{
Biopolitics and Labor Power. Virno reads
}

Foucault between Aristotle and Marx

ANDREA FAGIOLI (Dida

\section{Resumen}

El presente artículo se propone analizar la manera en que el filósofo italiano Paolo Virno piensa el concepto foucaulteano de biopolítica -que considera un corolario del concepto marxiano de fuerza de trabajo-, haciendo emerger la influencia que tiene en esa lectura no sólo Marx, sino también Aristóteles. En la primera parte abordaremos algunos trabajos de Michel Foucault de la primera mitad de los años 70 donde el filósofo francés sistematiza las nociones de "biopolítica de la población" y "anatomopolítica del cuerpo", de los cuales encontramos huellas en Virno, aún sin ser citados explícitamente por el autor. Posteriormente, nos centraremos en la peculiar lectura que Virno ofrece de los conceptos aristotélicos de potencia y acto, en particular por lo que concierne a la temporalidad de la potencia, en el marco de la cual el filósofo propone complicar el esquema binario aristotélico potencia-acto, introduciendo un tercer elemento: los actos potenciales. Finalmente, colocaremos esta reflexión en el horizonte marxiano, abordaremos el concepto de fuerza de trabajo y la escisión constitutiva que lo atraviesa, separando su dimensión estática de su dimensión dinámica (potencial). La hipótesis que intentaremos verificar es que la lectura de Aristóteles es clave para entender la manera en que Virno considera estrechamente imbricadas biopolítica y fuerza de trabajo.

Palabras clave: Virno. Biopolítica. Fuerza de trabajo. Aristóteles. Marx. Foucault.

\begin{abstract}
This paper aims to analyse the way in which the Italian philosopher Paolo Virno thinks the Foucauldian concept of biopolitics, that he considers only a corollary to the Marxian concept of labour-power, and I will try to shed light on the influence of not only Marx, but also Aristotle in his perspective. In the first part I will focus on some works that Michel Foucault wrote in the first half of the seventies, where he systematises the concepts of "biopolitics of the population" and

a Universidad Nacional de San Martín (UNSAM), Buenos Aires, Argentina. Doctor en Filosofía, e-mail: andrea.fagioli81@gmail.com
\end{abstract}


"anatomo-politics of the human body", even though Virno did not quote them directly. Subsequently, I will concentrate on the peculiar Virno's reading of the Aristotelian concepts of "potential" and "actual", in particular on the temporality of the potential; in fact Virno propounds to complicate the Aristotelian binary diagram potential-actual and he introduces a third element: the potential act. Finally, I will place these issues into the Marxian horizon, I will address the concept of labour power and the constitutive split that separates its static by its dynamic (potential) dimension. The hypothesis the I will try to verify is that the reading of Aristotle is a key issue to understand the way in which Virno considers deeply linked biopolitics and labour power.

Keywords: Virno. Biopolitics. Labor power. Aristotle. Marx. Foucault.

La noción foucaulteana de "biopolítica" ha dado lugar a un extenso debate a lo largo de las últimas décadas y lo ha hecho desde antes de la publicación (póstuma) de dos de los tres cursos (así llamados) "biopolíticos”, que el filósofo francés dictó en el Collège de France a finales de los años 70 (cf. FOUCAULT, 2007a; 2007b). En el ámbito italiano, por ejemplo, la lectura ante litteram ${ }^{2}$ de esa noción ha ocupado un lugar importante en trabajos teóricos "monumentales" (cf. SALINAS ARAYA, 2014), como los de G. Agamben, R. Esposito, M. Hardt y A. Negri, y también de algunos marxistas heréticos, como M. Lazzarato, "Bifo" o P. Virno (cf. FAGIOLI, 2016).

En el marco de ese debate, Paolo Virno ocupa una posición bastante lateral y parece dedicarse a la biopolítica casi “por obligación” o, dicho de otra manera, por la necesidad de tomar la palabra frente a una noción que se había vuelto, según el autor, "una moda". De hecho, en Gramática de la multitud, donde el registro oral de las clases ${ }^{3}$ le haya tal vez permitido una mayor libertad, leemos: “[e]n los últimos años, el concepto de 'biopolítica' se ha puesto de moda: se lo utiliza con frecuencia y para propósitos diversos. Sería importante evitar este empleo automático e irreflexivo" (VIRNO, 2003a, p. 84).

\footnotetext{
${ }^{1}$ El vocablo se debe, en realidad, al sueco R. Kjellen, quien lo acuñó para referirse al dinamismo de la vida en sociedad (cf. CASTRO, 2011), pero en el debate actual es usado exclusivamente en la acepción foucaulteana, que indica la emergencia, en el siglo XVIII, de un poder nuevo por el cual la vida de los hombres -y de las mujeres, agregamos- ha entrado en los cálculos políticos.

${ }^{2}$ La publicación en francés de Seguridad, territorio, población y Nacimiento de la biopolítica, en 2004, es posterior a esos "trabajos monumentales", que se han desarrollado autonómamente con respecto al trabajo de Foucault y que sólo parcialmente se han confrontado, posteriormente, con el texto foucaulteano.

${ }^{3}$ Gramática de la multitud es el resultado de un seminario dictado en el año 2001 en la Università della Calabria.
} 
La manera que propone para evitar lo que define "empleo automático e irreflexivo" consiste en inscribir el concepto de biopolítica en el horizonte marxiano, pensándolo como un corolario del concepto de "fuerza de trabajo", verdadero secreto del modo de producción capitalista. Desde este punto de vista, la biopolítica se coloca en un tejido de relaciones de fuerzas determinado histórica y geográficamente, en el marco del cual la vida que entra en los cálculos políticos es la vida de aquellos que producen para el capital.

Ahora bien. Virno sistematiza su perspectiva principalmente en dos textos: en El recuerdo del presente y en el ya citado Gramática de la multitud, escritos publicados, respectivamente, en 1999 y 2001 (ambos en 2003, en castellano). Hay que considerar, por lo tanto, que también en su caso se trata de una lectura que no toma en cuenta Seguridad, territorio, población y, sobre todo, Nacimiento de la biopolítica, que es el trabajo que más ha influenciado el debate biopolítico ${ }^{4}$.

Se trata, sin embargo, de una interpretación plenamente legítima, en la medida en que, como ha subrayado S. Chignola (2016), no es posible rastrear una manera unívoca en la cual la biopolítica se presenta en el trabajo de Foucault y que cada lector está obligado a plantearse el problema de qué significan "biopoder" y "biopolítica" en el conjunto de su producción ${ }^{5}$.

Si bien las referencias al trabajo de Foucault son escasas, casi nulas, nos parece posible afirmar que la perspectiva del "lector" Virno echa sus raíces principalmente en "Las mallas del poder", conferencia pronunciada por Foucault en Salvador de Bahía en 1976; en el primer curso "biopolítico" dictado en el Collège de France, y publicado en 1997 bajo el título de Defender la sociedad, y en el primer tomo de Historia de la sexualidad: La voluntad de saber.

En las páginas que siguen abordaremos la manera en que se presenta la biopolítica en aquellos textos foucaulteanos, para después enfocarnos en la lectura que Virno propone de Aristóteles y Marx que, como hemos intentado dejar en claro ya desde el título de este trabajo, es necesaria para entender a cabalidad el punto de vista del filósofo de Nápoles.

\footnotetext{
${ }^{4}$ Vale la pena recordar que en un ensayo publicado hace unos pocos años y titulado "El uso de la vida" (cf. VIRNO, 2017), al volver sobre la cuestión "biopolítica", el filósofo napolitano no toma en cuenta todo el material aparecido en casi tres lustros.

${ }^{5}$ Cabe aclarar que Virno no hace ninguna referencia al concepto de "biopoder", que en el trabajo de Foucault aparece sobre todo en el primer tomo de Historia de la sexualidad, quedando posteriormente al margen de su reflexión.
} 
Los objetivos que nos proponemos son principalmente dos: hacer emerger las huellas de lecturas foucaulteanas en el trabajo de Virno y, paralelamente, evidenciar la manera en que esta lectura nos ayuda a entender la especificidad de la forma en que el autor piensa el concepto de fuerza de trabajo.

Como es notorio, en el primer tomo de Historia de la sexualidad, en particular en el quinto y último capítulo, "Derecho de muerte y poder sobre la vida", Foucault plantea que entre los siglos XVII y XVIII tuvo lugar una inversión en la relación entre el poder y la vida. En este marco, sostiene que el viejo "derecho de vida y muerte" del soberano, se ha transformado. En aquellas páginas, muy leídas y comentadas, Foucault plantea que con ese sintagma se hace referencia a un derecho "asimétrico", en el sentido de que el soberano puede decidir quitar la vida al súbdito o no ejercer ese derecho, pero que la vida en sí no constituye más que un límite, el derecho último del poder de sustracción del rey. Por esto, Foucault reformula el sintagma con el cual indicar el derecho del soberano, definiéndolo como “derecho de hacer morir o de dejar vivir" (FOUCAULT, 2009, p. 128).

La introducción de la noción de biopolítica remite, justamente, a un giro radical en el ejercicio de ese derecho; un giro que el filósofo coloca entre los siglos XVII y XVIII y producto del cual el poder se ha tornado, en los términos de Foucault, un "derecho de hacer vivir o de arrojar a la muerte" (FOUCAULT, 2009, p. 130). Esta inversión en la lógica de funcionamiento del poder tiene, en la reflexión del francés, una relación profunda con el tipo histórico de sociedad en la cual esta lógica funciona y con el nacimiento del capitalismo. En otras palabras, en las sociedades precapitalistas "el poder era ante todo derecho de apropiación: de las cosas, del tiempo, de los cuerpos y finalmente de la vida”, es decir que "el poder se ejercía esencialmente como instancia de deducción, mecanismo de sustracción, derecho de apropiarse de una parte de las riquezas, extorsión de productos, de bienes, de servicios, de trabajo y de sangre, impuesto a los súbditos" (FOUCAULT, 2009, p. 128).

Según los análisis de Foucault, el sistema de poder que la monarquía había sabido organizar a partir del final de la Edad Media, presentaba dos grandes problemas para el desarrollo del capitalismo, en cuanto se trataba de mecanismos de poder poco eficientes y muy costosos. Por un lado "las mallas de la red eran demasiado grandes y un número casi infinito de cosas, de elementos, de conductas y de procesos escapaban al control del poder" 
(FOUCAULT, 1999, p. 242); por otra parte, “el poder era [...] esencialmente perceptor y predador. En esta medida, operaba siempre una sustracción económica y en consecuencia, lejos de favorecer y de estimular el flujo económico, era perpetuamente su obstáculo y su freno" (FOUCAULT, 1999, p. 234).

Para evitar peligrosas simplificaciones, nos parece necesario acá hacer dos salvedades. La primera es que los dos tipos de poder no son ordenados por Foucault en una secuencia cronológica, es decir que el biopoder no sustituye al poder soberano, sino que se articula con este y, de alguna manera, lo complementa (FOUCAULT, 2000). La segunda es que no queremos atribuir al filósofo una suerte de mecanicismo, producto del cual a un determinado tipo histórico de sociedad le pertenecería un determinado tipo de poder, según un esquema en el marco del cual el biopoder vendría a ser el poder específico de la sociedad capitalista $^{6}$. Al contrario, el biopoder aparece en los textos de Foucault como condición de posibilidad del nacimiento y del afianzamiento del modo de producción. Entonces, el surgimiento de una nueva lógica de funcionamiento del poder se debe a un cambio igualmente radical en el tipo histórico de sociedad del cual, paralelamente, fue conditio sine qua non.

Ahora bien. En La voluntad de saber, con el término biopolítica Foucault indica el momento en que el poder empieza a "producir fuerzas, a hacerlas crecer y a ordenarlas, más que a obstaculizarlas, doblegarlas o destruirlas" (FOUCAULT, 2009, p. 128). El poder pasa a administrar la vida, que entra, de esta manera, "en el dominio de los cálculos políticos explícitos” y, más en general, en la historia, en tanto blanco de técnicas políticas, aunque la vida "escapa de ellas sin cesar" (FOUCAULT, 2009, p. 135).

En trabajos como La voluntad de saber y Las mallas del poder, Foucault establece una división en el marco de lo que llama biopolítica con que individua dos polos alrededor de los cuales se ha transformado el poder en Occidente. Por un lado hace referencia a una anatomopolítica que se dirige al cuerpo del hombre-individuo y, por otro lado, a una biopolítica que se aplica a la población, en tanto hombre-especie. En el primer caso, trae a colación un conjunto de dispositivos isomorfos, como la cárcel, el hospital, el taller, la escuela, el cuartel, etcétera, que apuntan a la producción de un cuerpo productivo y dócil. Estos dispositivos tienen el objetivo de fijar al individuo a un determinado aparato

\footnotetext{
${ }^{6}$ Estamos conscientes de que la idea de "sociedad capitalista" en singular es igualmente problemática. Tómese acá como un elemento funcional a aclarar las "salvedades".
} 
productivo (FOUCAULT, 2001; 2016), teniendo su punto de culminación, a nuestro modo de ver, en la "disciplina” del sistema taylorista.

Por lo que concierne a la entrada de la vida en los cálculos políticos, en esos mismos textos Foucault hace referencia al interés del Estado centrado en cuatro ejes. El primero es la inquietud por la cuestión de la longevidad, la proporción entre nacimientos y decesos, abordada con las herramientas de las primeras demografías. El segundo es el interés por las enfermedades endémicas -más que las epidemias- consideradas en términos de costos económicos y como factor que se insinúa en la vida tornándola más débil, quitándole fuerzas. Medicina social e higiene pública son el resultado de esta inquietud. El tercer eje se desarrolla a partir de un "conjunto de fenómenos, de los cuales algunos son universales y otros accidentales", pero que no son eliminables completamente y que conllevan una serie de efectos al nivel de la "incapacidad, marginalización de los individuos, neutralización, etcétera" (FOUCAULT, 2000, p. 221). El autor se refiere, por un lado, al problema de los accidentes, las enfermedades, por otro a problemas como la vejez que expulsan al individuo del campo de las capacidades y de las actividades. La biopolítica consistirá, en este tercer caso, en el armado de instituciones de asistencia, que -agregamos nosotros- tendrán su apogeo con el sistema de Estado de bienestar keynesiano. El último eje remite a la intervención en el ambiente de existencia, tanto en términos "naturales", en el sentido geográfico, climático e hidrográfico, como en términos de ciudades.

Ahora bien. Como hemos adelantado en los párrafos introductivos, Virno pone la "biopolítica" en relación con el concepto de fuerza de trabajo. En los dos textos que tomamos como base para nuestro análisis encontramos dos fórmulas similares que afirman derechamente este vínculo hipotetizado por el autor. En El recuerdo del presente el autor escribe: "[e]l concepto de fuerza de trabajo, recorriendo todo giro de frases en los análisis económicos y sociológicos, ha quedado casi impensado. Los filósofos profesionales lo descuidan alzándose de hombros, ocupándose totalmente de temas que son sólo un corolario de aquel" (VIRNO, 2003b, p. 68). En Gramática de la multitud encontramos palabras más polémicas todavía:

para comprender el nudo racional del término "biopolítica", es preciso partir de un concepto distinto, acaso más complicado desde el punto de vista filosófico: el concepto de fuerza de trabajo. Se habla mucho de esta noción en las ciencias sociales, aunque demasiadas veces se deja de lado su carácter áspero y paradojal. Si los filósofos de profesión quisieran ocuparse de un tema serio, harían bien en dedicarle esfuerzo y atención a la noción de fuerza de trabajo. ¿Qué significa "fuerza de trabajo"? Significa potencia para producir. Potencia, es decir, facultad, 
capacidad, dynamis. Potencia genérica, indeterminada [...] La fuerza de trabajo encarna (literalmente) una categoría fundamental del pensamiento filosófico: la potencia, la dynamis (VIRNO, 2003a, pp. 85-86).

Un segundo elemento que emerge es que este abordaje de la cuestión y el léxico usado por Virno remiten claramente, más allá del ámbito del marxismo, al horizonte conceptual aristotélico ${ }^{7}$. En los próximos apartados, por lo tanto, nos enfocaremos en la peculiar lectura de Aristóteles que propone nuestro autor, en particular por lo que concierne a la cuestión de la temporalidad de la potencia.

En su polémica contra Euclides de Megara y sus discípulos, quienes sostenían la simultaneidad de potencia y acto, que eliminaba -de hecho- la diferencia entre los dos términos, Aristóteles plantea "la distinción estructural [...] entre el ser en potencia y el ser en acto" (REALE, 1999, p. 173), sugiriendo que "cabe que algo pueda ser, pero no sea, y pueda no ser, pero sea" (ARISTÓTELES, 1994, p. 369). En este sentido el Estagirita postula la anterioridad del acto con respecto a la potencia según el concepto (to lógo), según el tiempo (to crono) y según la sustancia (to ousía $)^{8}$.

Siguiendo la explicación esquemática de un importante comentarista de Aristóteles, como G. Reale (1999), podemos afirmar que "el acto es anterior a la potencia en cuanto al concepto, porque la potencia sólo puede definirse previo conocimiento del acto de que es potencia” (p. 175) y que por esto la condiciona. Por lo que concierne al tiempo la cuestión es parcialmente más complicada, ya que, si consideramos un ente o individuo particulares, encontramos que este existe antes en potencia y, después, en acto. Sin embargo, el razonamiento no se centra en entes o individuos particulares. Escribe el Estagirita:

lo que es en acto se genera siempre de lo que es en potencia por la acción de algo que es en acto, por ejemplo, un hombre por la acción de un hombre, un músico por la acción de un músico, habiendo siempre algo que produce el inicio del movimiento. Y lo que produce movimiento está ya en acto (ARISTÓTELES, 1994, p. 382).

\footnotetext{
${ }^{7}$ En realidad, la tematización filosófica del vocablo dynamis es anterior a Aristóteles y "se encuentra primeramente en relación con la naturaleza, phýsis, en los textos de los primeros filósofos y también en Platón" (DEZZUTTO, 2010). Sin embargo, estas cuestiones exceden por completo los objetivos de este trabajo.

${ }^{8}$ Virno (2003b) usará "noción", en lugar de "concepto" y "esencia" en vez de "sustancia".
} 
Decimos entonces que "[e]l acto es anterior a la potencia en cuanto al tiempo, porque lo que se engendra y llega a ser requiere de una causa eficiente ya en acto" (REALE, 1999, p. 176). Finalmente, el acto es anterior a la potencia en cuanto a la sustancia (o esencia o entidad ${ }^{9}$ ) porque

las cosas que son posteriores en cuanto a la generación son anteriores en cuanto a la forma específica [...] (así el adulto es anterior al niño, y el hombre al esperma; pues lo uno posee ya la forma específica y lo otro no) (ARISTÓTELES, 1994, p. 383).

Además -agrega el Aristóteles- el acto es anterior a la potencia en cuanto a la entidad "porque todo lo que se genera progresa hacia un principio, es decir hacia un fin [...] y el acto es fin, y la potencia se considera tal en función de él", es decir que "se posee el arte de construir para construir, y la capacidad de teorizar para teorizar, pero no se teoriza para tener la capacidad de teorizar" (ARISTÓTELES, 1994, p. 383).

Ahora bien. Virno invierte el punto de vista aristotélico en cuanto a la organización entorno al eje antes-después de potencia y acto y lo hace a partir de lo que llama "acepción antimegárica de simultaneidad". En base a esta, nuestro autor comparte la afirmación megárica de que "[e]s la potencia sólo cuando es el acto", pero "a condición de agregarle una pequeña cláusula: 'en el tiempo". Es decir que, para Virno, "[1]a potencia existe en el tiempo sólo cuando es el acto" (VIRNO, 2003b, p. 47).

Para Aristóteles, como es notorio, potencia y acto se encuentran no sólo en el origen del movimiento, sino que tienen también un papel fundamental en su concepción del tiempo, ya que no podría haber tiempo si el "ser potencial" y el "ser actual" coincidieran. Esquivando la alternativa con respecto al hecho de si potencia y acto son conceptos temporalizados o temporalizantes, es decir si se refieren a la sucesión cronológica o al orden temporal, Virno plantea que la potencia representa el pasado no-cronológico del acto, un pasadopotencia que es irrealizable y que puede ser pensado, recalcando la diferencia introducida por É. Benveniste (1997), como la facultad de lenguaje con respecto a cualquier enunciado producido por el sujeto hablante.

Vayamos por partes. Nuestro autor sostiene que lo que cae siempre en el tiempo y que siempre puede ser objeto de percepción es el acto. Al contrario, la potencia no cae en el tiempo, sino que constituye una perenne inactualidad. Con este sintagma, quiere indicar que:

\footnotetext{
9 "Entidad" es el vocablo que usa T. Calvo Martínez en su traducción por Gredos que usamos aquí.
} 
"[s]i el acto es el 'ahora', la potencia es el 'siempre'; lábil el primero, permanente la segunda" (VIRNO, 2003b, p. 31). La relación entre potencia y acto se configura, entonces, en la reflexión de nuestro autor, como la relación entre un "no-ahora" perenne y un "ahora" particular. Lo que se realiza, desde este punto de vista, no es la potencia -que es constitutivamente irrealizable- sino un acto potencial que, en tanto tal, puede realizarse, pero también puede no realizarse. Sin embargo, decir "acto potencial" no equivale a decir potencia, así como el acto no es una "potencia actuada". Retomando el paralelismo con Benveniste, Virno estructura su razonamiento sobre tres niveles:

(a) la facultad del lenguaje, es decir, el simple y puro poder-decir, la disposición genérica a significar y a comunicar; (b) una o más enunciaciones virtuales, como por ejemplo: las frases amorosas anidadas en los meandros de la lengua; (c) el acto de la palabra, siempre único e irrepetible, que realiza esta o aquella enunciación virtual (VIRNO, 2003b, p. 36).

Y a partir de esta distinción, plantea su posición sobre lo que considera una equivocación, que echa sus raíces en la falta de comprensión de la facultad de lenguaje. Continúa el autor:

[p]ues bien, el pasaje de (b) a (c) no tiene nada que ver con la relación entre potencia y acto: se tiende a pensar lo contrario sólo porque se descuida o se entiende mal el peculiar estatuto de (a). Tanto (b) como (c) ostentan, aunque con distinta intensidad, el modo de ser de la presencia: el acto real es un «ahora», el potencial un casi-ahora. El modo de ser de la facultad, por su lado, está marcado por una duradera inactualidad. Mientras (b) posee la estructura de un evento actual, aunque no existe ahora, (a) existe seguramente, pero permanece no-presente. La potencia se distingue de una acción eventual no menos que de una efectiva; (a) se opone de igual modo a (b) y (c) (VIRNO, 2003b, p. 36).

Virno afirma que podríamos hablar de una anterioridad del acto por sobre la potencia a nivel lógico, ontológico y cronológico, sólo en la medida en que pensemos la potencia en tanto acto potencial, pero no es así si la pensamos como un permanente no-ahora. La tesis del filósofo de Nápoles toma forma a partir de un cortocircuito entre orden temporal y sucesión cronológica, en base al cual el no-ahora permanente de la potencia y el ahora del acto son caracterizados, a la vez, por una diacronía y por una concomitancia. Es decir que en base al orden temporal la potencia es anterior al acto, aunque se trate de un antes sin fecha que no tiene nada en común con la sucesión empírica de dos acontecimientos. Por otro lado, en lo que concierne a la sucesión cronológica "potencia y acto son siempre simultáneos: la potencia no es la prefiguración del acto, sino su correlato heterogéneo, su sombra inconmensurable" (VIRNO, 2003b, p. 45). 
Veamos más en detalle. En primer lugar, Virno postula que la potencia es anterior al acto en cuanto al tiempo, es decir en cuanto al orden temporal, y lo hace remitiendo nuevamente a Benveniste. El lingüista francés plantea, al analizar la naturaleza de los pronombres, que el papel de los deícticos es el de "ofrecer el instrumento de una conversión, que puede denominarse la conversión de un lenguaje en discurso" (BENVENISTE, 1997, p. 175); a partir de ahí, Virno sostiene que esta conversión remite a una diferencia de naturaleza que indica dos planes temporales distintos. Es decir que las partes del discurso que señalan la actualidad -el ser aquí y ahora de un enunciado-, representan el pasaje de la pura posibilidad de decir -o sea un pasado en el cual existe solamente la posibilidad de la lengua- al discurso presente. En este sentido, el filósofo plantea la existencia de dos pasados de un acto: por una parte este pasado no-cronológico y por otra parte las antiguas actualidades que han precedido en el tiempo (y que entran en una secuencia causal) del acto. Esto significa, por ejemplo, que el pasado de la Revolución haitiana es tanto el conjunto de acontecimientos que han llevado a los esclavos negros a sublevarse y a derrotar al ejército colonial, como la posibilidad del ser humano de poner en cuestión los a priori históricos que subyacen a su modo de existencia.

Para concluir. En la refinada elaboración teórica de Virno, la potencia no cae en el tiempo y subsiste también en ausencia de un acto, sin embargo, en este caso permanece fuera de la sucesión cronológica. Pero la apuesta de Virno a la que hemos aludido consiste en plantear que la potencia existe en el tiempo sólo cuando es acompañada, y señalada, por el acto y esto se vincula con el tema al cual nos dedicaremos en el apartado que viene: la fuerza de trabajo. De hecho, el autor sugiere que nosotros hacemos experiencia de la fuerza de trabajo durante la realización de un trabajo particular, que la pone en "entredicho", pero hacemos experiencia de ella como de algo que permanece y no muta. Virno pone así a su "Aristóteles invertido" en el corazón de su análisis del modo de producción capitalista, llevándolo a lo que Marx llama: “oculta sede de la producción” (MARX, 2010, p. 214).

Hemos dicho que la fuerza de trabajo es, para Virno, el concepto clave para entender la biopolítica. Vayamos, entonces, al origen de este concepto y veamos la definición que 
nos propone Marx, quien lo aborda desde dos puntos de vista distintos. En primera instancia leemos en El Capital:

por fuerza de trabajo o capacidad de trabajo entendemos el conjunto de las facultades físicas y mentales que existen en la corporeidad, es decir, en la personalidad viva de un ser humano y que él pone en movimiento cuando produce valores de uso de cualquier índole (MARX, 2010, p. 203).

En este sentido, nos parece importante retener el vocablo "facultades", en que resuenan los problemas que hemos abordado en el apartado anterior. De hecho, el propio Marx inserta la cuestión en la senda aristotélica, cuando escribe: "[q]uien dice capacidad de trabajo no dice trabajo, del mismo modo que quien dice capacidad de digerir no dice digestión” (MARX, 2010, p. 211).

Paralelamente, el filósofo de Tréviris indica que la fuerza de trabajo, en el marco del modo de producción capitalista, se constituye como mercancía ${ }^{10}$. Sin embargo, como ha sido subrayado, se trata de una mercancía que tiene una peculiaridad, es decir que no se agota con el uso, sino que su uso da al capitalista más valor del que ha recibido el trabajador, y más del que el capitalista ha pagado (cf. THOMAS, 2010). Con el vocabulario del apartado anterior diríamos que la fuerza de trabajo en tanto potencia (social -recordémoslo- y no individual) es perenne inactualidad y, por lo tanto, no se agota. Al contrario es "convertida" en trabajo -en un conjunto de actos que caen en el tiempo-, produciendo más valor del que recibe el poseedor individual de fuerza de trabajo.

Ahora bien. En el debate contemporáneo, el francés P. Macherey (2012) ha usado dos términos alemanes distintos que, aunque no sea posible rastrearlos en el mismo sentido en la obra marxiana, nos parecen muy sugerentes para entender lo "mágico" de la producción de valor en el modo de producción capitalista (en particular por lo que concierne al modelo industrial) y para entender la apuesta conceptual de Virno. Estos dos términos, que escinden el concepto de fuerza de trabajo son Arbeitskraft y Arbeitsvermögen.

\footnotetext{
${ }^{10}$ En la constitución de este mercado entran en juego dinámicas violentas, que Marx (2010) ha descrito en términos de acumulación originaria, ya que el obrero vende el conjunto de facultades físicas y mentales de las que dispone sólo en la medida en que ha sido privado de los medios de producción. Esa doble liberación a la que se refiere Marx (1989) en los Gründrisse: de las relaciones personales de sumisión y de los medios de producción, hace que una potencia como la genérica facultad de producir se torne un bien en sí y que el obrero no sólo pueda, sino que esté obligado a venderla porque no puede aplicarla solo.
} 
Con el primero el filósofo francés remite a la dimensión estática de la fuerza de trabajo, lo que ya es y que el capitalista paga, reservándose el derecho de usarla. Al "uso" se refiere la Arbeitsvermögen, fuerza de trabajo en sentido dinámico, es decir potencia. Retomando la distinción propuesta por D. Deleule y F. Guery (1972), entre fuerza productora y fuerza productiva, Macherey postula que el capital compra con el salario la fuerza productora, es decir la Arbeitskraft, en tanto valor determinado (histórica y geográficamente) por la reproducción de la fuerza de trabajo. Se trata, como afirma R. Ciccarelli (2018), de una fuerza que es "privada de los caracteres específicos de la "personalidad viviente" y "sometida a las normas que permiten evaluar, calibrar y moldear su uso" (p. 86). Sin embargo, lo que usa es la fuerza productiva, una potencia que puede ser -más bien tiene que ser- moldeada.

Lo que, según nosotros, constituye la especificidad de la lectura de la Metafísica de Aristóteles propuesta por Virno, -en particular la complicación del esquema binario clásico potencia-acto, y en la inclusión de un tercer elemento: los actos potenciales-, permite ahondar no sólo en la concepción de biopolítica de Virno, sino que, gracias a la distinción Arbeitskraft/Arbeitsvermögen, podemos empujar esa interpretación hasta más allá del punto donde la lleva el propio autor.

Veamos más en detalle. Virno sostiene que "la biopolítica es sólo un efecto, una reverberación, una articulación de aquel hecho primario -histórico y filosófico al mismo tiempo- que consiste en la compraventa de la potencia en cuanto potencia” (VIRNO, 2003a, p. 89). En este sentido, la necesidad del capital de comprar la única mercancía cuyo consumo produce (plus)valor, hace que surja el interés por "el receptáculo del cual ella es indistinguible, o sea el cuerpo viviente; más aún lo pone a la vista en tanto objeto y blanco de innumerables y diferenciadas estrategias de control” (VIRNO, 2003a, p. 89). Es decir que, "el cuerpo viviente se convierte en objeto a gobernar no tanto por su valor intrínseco, sino porque es el sustrato de la única cosa que verdaderamente importa: la fuerza de trabajo como suma de las más diversas facultades humanas” (VIRNO, 2003a, p. 87).

Encontramos en estos pasajes una concepción de biopolítica que, aún sin alusiones al texto de Foucault, parece tomada literalmente de La voluntad de saber. En la definición de biopolítica en tanto gobierno y administración de "la vida en cuanto tal, la vida como mero proceso biológico" (VIRNO, 2003a, p. 88), resuena lo que Foucault (2009) llama "biopolítica de la población” y que se dirige al cuerpo del hombre-especie; al contrario, en 
la referencia al cuerpo como objeto y blanco de estrategias de control aparece evidente la alusión a la noción de anatomopolítica del cuerpo del hombre-individuo.

Si pensamos, en este marco, la tripartición potencia/acto potencial/acto propuesta por Virno, desde nuestro punto de vista biopolítica y anatomopolítica se inscriben en el espacio que se coloca entre la potencia y los actos potenciales, sin obviamente saturarlo, porque allí entran en juego también otros elementos de carácter conflictivo (léase lucha de clases), culturales, etc. Pero lo que nos interesa aquí es que lo que el capital adquiere como mercancía es potencia para producir, una potencia que caracteriza genéricamente al bomo sapiens en tanto ser inespecializado ${ }^{11}$. A la dimensión reproductiva y de salud pública de la población (como tabernáculo de la Arbeitskraft) se dirige, en modos distintos dependiendo del contexto histórico y geográfico, la biopolítica. Pero esta potencia tiene que ser moldeada, fijada a un aparato productivo, es decir que tiene que incorporar una serie de habilidades técnicas necesarias para trabajar con ciertos tipos de máquinas. Dicho en otras palabras, la fábrica no podría existir sin la posibilidad de actualizar un conjunto de actos potenciales.

Entonces nos parece que biopolítica y anatomopolítica se insertan en el terreno de la potencia, funcionando como elementos que -dicho en términos de Benveniste- la "convierten" de algo constitutivamente inactuable en una mercancía y la predisponen para su uso, actuando sobre el cuerpo del hombre-especie y el cuerpo del hombre individuo en las maneras que recuerda Foucault (2001; 2009; 2016).

Ahora bien. Si esta reflexión parece referirse exclusivamente a las sociedades disciplinarias y al capitalismo industrial, por lo menos en cuanto concierne a la dimensión anatomopolítica, cabe preguntarse qué ocurre con el pasaje a lo que Virno llama "postfordismo". Tratemos, para cerrar, de esbozar brevemente una hipótesis, cuyo desarrollo necesitaría un artículo aparte ${ }^{12}$. En Gramática de la multitud el filósofo sostiene dicho de manera muy esquemática-, que en el capitalismo contemporáneo el lenguaje

\footnotetext{
${ }^{11}$ Virno retoma la concepción antropológica de ser carencial e inespecializado, y por lo tanto potencial, tal y como se presenta en cierta antropología filosófica alemana del siglo XX (cf. GEHLEN, 1987).

${ }^{12}$ Por otra parte, vemos que el terreno reproductivo del welfare se abrió a la explotación capitalista, obligando a las ciudadanas y a los ciudadanos a hacerse cargo de su propia condición, según una lógica costo-beneficio, en ámbitos de los que, hasta hace unas pocas décadas, el Estado era el responsable único. Pero, como hemos dicho, sería muy extenso desarrollar estas cuestiones aquí.
} 
irrumpe en el ámbito de la producción y lo que es una facultad se vuelve un recurso productivo directo.

Al trabajador no se le pide -escribe- un cierto número de frases standard, sino una actividad comunicativa informal, dúctil, que le permita hacer frente a las más diversas eventualidades [...] En términos de filosofía del lenguaje, diré que lo que se pone en movimiento aquí no es la parole sino la langue; la propia facultad de lenguaje, no una específica aplicación (VIRNO, 2003a, p. 101).

De esta manera, parecen desaparecer la importancia de los "actos potenciales" y, paralelamente, parece que la potencia, cuyo paralelo con la langue Virno subraya, pueda ser puesta a trabajar directamente. Sin embargo, si bien es cierto que la reflexión sobre la Metafísica de Aristóteles parece quedar de lado, hay que colocar este planteamiento en el marco del análisis específico del modelo postfordista que propone Virno. En este sentido, lo que es necesario tener en cuenta, es que cuando el saber -general intellect en los términos marxianos usados por Virno- se vuelve un recurso productivo directo, el trabajo vivo (es decir, el trabajador) incorpora parte de lo que antaño pertenecía al capital fijo, es decir, a las máquinas. De esta manera, más que de una anatomopolítica impuesta desde afuera, es necesario pensar en términos de "uso de la vida", para referirse a la conversión de la potencia, siendo el uso de la vida una prerrogativa del animal humano que, lejos de ser natural, "requiere adiestramiento, ejercicios prolongados, procedimientos experimentales, adquisición de técnicas, observancia de reglas" (VIRNO, 2017, p. 137). Y es justamente sobre el terreno de la manera en que se usa la vida, más que de la resistencia a la disciplina, que un autor como Virno, que remite directamente y sin remordimiento a Marx, inscribe hoy la lucha de clases que, a lo largo del capitalismo industrial, se colocaba en la fábrica.

\section{Bibliografía}

ARISTÓTELES. Metafisica. Madrid: Gredos, 1994.

BENVENISTE, É. Problemas de lingüistica general (vol. 1). Ciudad de México: Siglo XXI, 1997.

CASTRO, E. Lecturas foucaulteanas, Una historia conceptual de la biopolítica. La Plata: Unipe, 2011. 
CHIGNOLA, S. 2000 D.C. Biopotere e biopolitica: sulle tracce della discussione. Euronomade, 13 de junio de 2016. Disponible en: http://www.euronomade.info/?p=7351. Último acceso 21 de marzo de 2019.

CICCARELLI, R. Forza lavoro. Il lato oscuro della rivoluzione digitale. Roma: DeriveApprodi, 2018.

DELEULE, D. y GUERY, F. Le corp productif. Paris: Mame, 1972.

DEZZUTTO, F. “Dynamis y causalidad en Aristóteles y Plotino”. Santa Fe: XXI Symposio Nacional de Estudios Clásicos, 2010.

FAGIOLI, A. Política y vida. Perspectivas posoperaístas. Pléyade. Revista de Humanidades y Ciencias Sociales, 17, 2016, pp. 155-180.

FOUCAULT, M. Estética, ética y hermenéutica. Obras esenciales volumen III. (vol. 3). Barcelona: Paidós, 1999.

FOUCAULT, M. Defender la sociedad. Curso en el Collège de France (1975-1976). Buenos Aires: Fondo de Cultura Económica, 2000.

FOUCAULT, M. Vigilary castigar. Nacimiento de la prisión. Buenos Aires: Siglo XXI, 2001.

FOUCAULT, M. Nacimiento de la biopolítica. Curso en el Collège de France (1978-1979). Buenos Aires: Fondo de Cultura Económica, 2007a.

FOUCAULT, M. Seguridad, territorio, población. Curso en el Collège de France (1977-1978). Buenos Aires: Fondo de Cultura Económica, 2007b.

FOUCAULT, M. Historia de la sexualidad I. La voluntad de saber. Buenos Aires: Siglo XXI, 2009.

FOUCAULT, M. La sociedad punitiva. Curso en el Collège de France (1972-1973). Buenos Aires: Fondo de Cultura Económica, 2016.

GEHLEN, A. El hombre. Su naturaleza y su puesto en el mundo. Salamanca: Sígueme, 1987.

MACHEREY, P. "Le sujet productif." Stage de formation des professeurs de Philosophie, Lille, 10 de mayo de 2012. Disponible en: https://philolarge.hypotheses.org/1245. Último acceso 25 de noviembre de 2018.

MARX, K. Elementos fundamentales para la crítica de la economía política: borrador (vol. 1). Ciudad de México: Siglo XXI, 1989.

MARX, K. El capital. Crítica de la economía politica (vol. 1). Buenos Aires: Siglo XXI, 2010.

REALE, G. Guía de lectura de la "Metafísica” de Aristóteles. Barcelona: Herder, 1999.

SALINAS ARAYA, A. La semántica biopolítica: Foucault y sus recepciones. Viña del Mar: Cenalties, 2014.

THOMAS, P. Labour-power. Krisis. Journal of Contemporary Philosophy, vol. 2, pp. 49-54, 2010.

VIRNO, P. Gramática de la multitud. Para un análisis de las formas de vida contemporaneas. Buenos Aires: Colihue, 2003a. 
VIRNO, P. El recuerdo del presente. Ensayo sobre el tiempo histórico. Buenos Aires: Paidós, 2003b.

VIRNO, P. La idea de mundo. Intelecto público y uso de la vida. Buenos Aires: La Marca, 2017.

RECIBIDO: 08/04/2019

RECEIVED: 04/08/2019

APROBADO: 02/07/2020

APPROVED: 07/02/2020 